\title{
Health, city, and urban design
}

\author{
Mahyar Arefi ${ }^{1} \cdot$ Noha Nasser ${ }^{2}$
}

Accepted: 7 May 2021 / Published online: 21 May 2021

(c) The Author(s), under exclusive licence to Springer Nature Limited 2021

With the COVID-19 Pandemic rampaging the entire world for over a year now, we are experiencing unpredictable circumstances, the likes of which humanity has rarely seen in our lifetime. Without trying to rehash the business as usual about how the Pandemic has transformed our lives the way we knew it, this editorial piece outlines the following articles that, one way or another, shed light on how some of these new experiences have affected thinking about urban design.

But before introducing these new contributions, the commonalities on the terminologies and concepts characterizing them seem both inevitable and interesting. Obviously "health," "healthy city," or the "public health and the city" come to mind as the high-priority themes overshadowing other possible verbiage. Health in the city, town, or the neighborhood emerge as variations on a theme. Other terms including resiliency, satisfaction, and the public space also seem logical in situations where people ought to obey protocols, i.e., social distancing in the public space.

Against this broad background, the first article by Julian Martins explores "work in and for a healthy city" by investigating the interplay among its three key facets. Urban designers-she argues-ought to enhance the quality of public space through "sociability," and also, mixing productive/economic activities, i.e., the manufacturing and industrial uses within the context of the flows of goods and people. Second, places of work-she recommends-should accommodate not only different workspaces but also diversify accessibility and community patterns through new workspace and working practices, i.e., the potential demise or falling from favor of typical office towers that might even affect housing design as well. Third, work/economy in place could significantly affect retail/business locations and eventually neighborhood and town centers as we know them.

\footnotetext{
Mahyar Arefi

mahyararefi@gmail.com

Jundi-Shapur University of Technology, Dezful, Iran

2 Cardiff University, Cardiff, UK
}

Keeping them vital could entail changing the relationship between streets, public spaces, and building typologies.

In the second article on "informal urbanism in the state of uncertainty" Kamalipour and Peimani explore how dealing with a Pandemic during the states of emergencies brings to mind informal urbanism as yet another aspect of thinking about healthy cities or health in the city. Coping with social isolation, lockdown, or curfews exemplifies three types of unexpected situations during unpredictable circumstances. The authors offer a "nuanced understanding of informality," which might apply across a broad array of urbanisms from the formal to informal. Revisiting the "assemblage theory," authors question the formal-informal binary distinction, and instead, call for a broader array of urban interventions, not only limited to street vending and informal settlements, but also different modes of transportation. Authors also distinguish between the global "South" and "North" on one hand, and the "temporal" vs "tactical" interventions as yet another meaningful caveat on the other. Reflecting on the interface between urban design, health, and informal urbanism, the authors also recommend to adopt a nuanced understanding of different forms of informal urbanism, instead of taking a reductionist approach by preconceived notions of what forms do or do not work in the formal-informal urban design dichotomy.

In the third article on "antifragile urban forms," Sartorio et al., raise similar concerns about the new experience in the wake of a pandemic by ask specific questions on how urban designers can deal with unexpected public health transformations. They specifically revisit resiliency as a terminology familiar with most planners and designers, and redefine and characterize its three newly unfolding traits, namely, multiscalar, evolutionary, and spatiality as a complex set of budding and promising research agenda that can set in gear how a constantly growing pandemic can morph into what authors call an "antifragility" that "disentangles issues of practiceready value without losing sight of urban complexity."

In the fourth article "COVID-19 Pandemic and Public Space," Sepe sheds light on yet another aspect of the pandemic-public space interface. More specifically, she 
conducts research on a wide range of the term public space from waterfronts, gardens, and parks to nature paths and squares. This research pays more attention to the flexibility of these types of public spaces under the COVID-19 breakout. Beyond some general modifications to their routine usage, her findings underline some updates including close ties between citizens' adaptability at high risk and the emergency situations, which, in her opinion, suggests how public spaces can become livable instead of categorically preventing their access to these at-risk publics.

In the fifth article on "the influence of location, planning and design features on residents' satisfaction with security in public housing," Jegede et al., address and investigate the nexus between residents' satisfaction and location, planning, and public housing. Their findings highlight how territoriality and defensible space, encouraging communal living, enhancing openness, and natural surveillance along with the effective use of architectural features, overall, increase secured settings. Creating better home environs, in turn, deter or disrupt criminal activities. Thus, achieving these features ensure resident satisfaction of their collective domiciles.

Since among other things, COVID-19 has negatively affected tourism worldwide, exploring potential linkages between tourists' satisfaction and urban design has also attracted new attention. The sixth article on elements that affect "foreign tourists' satisfaction" addresses this fundamental question. While the public health component of the public space plays pivotal roles in creating or preserving healthier cities and citizens, satisfaction invokes another important word in the urban design lingo. Ryu and Kwon focus on the tourists' satisfaction and its public and urban design policy implications. Having Seoul, Korea as their case study, the authors administered a survey questionnaire among 250 foreign tourists. As it turns out, cityscape plays key roles in satisfying the tourists compared to other competing variables including shopping, security, transportation. This somewhat surprising finding has much to do with the fact that while tourism accelerates homogeneity, creating attractive cityscapes, influences the tourists more than coming to get on the shopping spree per se. We hope that these six articles provide some new understandings about urban designers' roles and responsibilities in being hopeful and vigilant while new solutions emerge in times of uncertainly.

Publisher's Note Springer Nature remains neutral with regard to jurisdictional claims in published maps and institutional affiliations. 